\title{
Upregulation of heme oxygenase-1 in Kupffer cells blocks mast cell degranulation and inhibits dendritic cell migration in vitro
}

\author{
YUAN-YUAN MA ${ }^{1 *}$, MU-QING YANG ${ }^{2 *}$, ZHI-GANG HE ${ }^{2}$, \\ MAO-HONG FAN ${ }^{3}$, MAN HUANG $^{4}$, FEI TENG ${ }^{4}$, QING WEI ${ }^{1}$ and JI-YU LI ${ }^{2}$
}

Departments of ${ }^{1}$ Pathology and ${ }^{2}$ General Surgery, Shanghai Tenth People's Hospital of Tong Ji University, Shanghai 200072, P.R. China; ${ }^{3}$ Department of Chemical and Petroleum Engineering, University of Wyoming, Laramie, WY 82071, USA; ${ }^{4}$ Department of Good Clinical Practice, Shanghai Tenth People's Hospital of Tong Ji University, Shanghai 200072, P.R. China

Received March 22, 2016; Accepted November 30, 2016

DOI: $10.3892 / \mathrm{mmr} .2017 .6448$

\begin{abstract}
Kupffer cells (KCs) influence liver allografts by interacting with other non-parenchymal cells. However, the exact mechanism remains unclear. Upregulation of heme oxygenase-1 (HO-1) in KCs upon interaction with mast cells (MCs), and the effects on dendritic cell (DC) function, were investigated in the present study. $\mathrm{KCs}, \mathrm{MCs}$ and $\mathrm{DCs}$ were prepared from 8-10-week-old C57BL/6 mice. KCs were pretreated with PBS, dimethyl sulfoxide, hemin (50 $\mu \mathrm{M}$; HO-1 inducer), and zinc protoporphyrin (50 $\mu \mathrm{M}$; HO-1 inhibitor) for $8 \mathrm{~h}$. Reverse transcription-polymerase chain reaction and western blotting was performed to determine HO-1 mRNA and protein levels in $\mathrm{KCs}$, respectively. C-C motif chemokine receptor 7 (CCR7) surface molecules were measured using flow cytometry, and prostaglandin E2 (PGE2), C-C motif chemokine ligand (CCL) 19 and CCL21 were measured by ELISA. The Transwell model was used to investigate the migration of DCs. Pretreatment of KCs with hemin induced HO-1 transcription and protein expression, and interacted with and stabilized MC membranes. When co-cultured with MCs, the expression of CCR7 on DCs was reduced, and PGE2, CCL19 and CCL21 were similarly decreased. DC migration was also impaired. Upregulation of HO-1 in KCs blocked MC degranulation and reduced DC migration.
\end{abstract}

Correspondence to: Professor Ji-Yu Li, Department of General Surgery, Shanghai Tenth People's Hospital of Tong Ji University, 301 Yanchang Road, Shanghai 200072, P.R. China

E-mail: leejiyu@sina.com

Professor Qing Wei, Department of Pathology, Shanghai Tenth People's Hospital of Tong Ji University, 301 Yanchang Road, Shanghai 200072, P.R. China

E-mail: weiqing1971@aliyun.com

*Contributed equally

Key words: Kupffer cell, heme oxygenase-1, mast cell, dendritic cell, migration

\section{Introduction}

Heme oxygenase-1 (HO-1) has an important protective role in various disease models due to its anti-inflammatory, anti-apoptotic and anti-proliferative actions $(1,2)$. HO-1 also has an important role in the allograft immune response. Following liver transplantation, various cell types induce HO-1 overexpression to prevent ischemia reperfusion injury (IRI) and immune rejection (3-5). It is well established that Kupffer cells (KCs) are among the most important resident macrophages of the liver and account for $\sim 20 \%$ of all hepatic non-parenchymal cells (6). Increased attention has focused on the potential roles and mechanisms of $\mathrm{KCs}$ in tolerance induction following liver allografts. Wang et al (7) demonstrated that preconditioning with nodosin perfusion induced $\mathrm{HO}-1$ expression in $\mathrm{KCs}$ following transplantation, and this upregulation was demonstrated to be protective against IRI, a process which is thought to facilitate immune rejection.

It is now appreciated that the function of mast cells (MCs) is not limited to allergic disease or chronic immune rejection. Recent studies have reported that active MCs degranulate to induce IRI and acute immune rejection $(8,9)$, and these cells influence the tissue microenvironment via release of a variety of pre-existing and cell-synthesized mediators, including proteases, cytokines, chemokines and arachidonic acid metabolites (10). A previous study reported that MC degranulation may disrupt peripheral immune tolerance and result in immune rejection (11), and also suggests that MC degranulation may promote IRI in the rat liver (12). Stabilizing MC membranes may, therefore, alleviate immune rejection and IRI. Takamiya et al (13) demonstrated that HO-1 stabilizes MCs following exposure to the anti-inflammatory compound bilirubin.

Dendritic cells (DCs) are one of the most potent types of antigen-presenting cells and are known to be important in triggering immunity to various types of antigens (14). Under normal circumstances, DCs are immature in vivo, and co-stimulation of CD80, CD86 and major histocompatibility complex class II at the surface of DCs is low (15). Immature DCs migrate into secondary lymphoid organs and differentiate into mature DCs that are capable of triggering immune rejection following transplantation. DCs express C-C motif chemokine receptor 1 
(CCR1), CCR7, CCR5 and CCR6 chemokine receptors, and exhibit chemotaxis (16). Preventing DC migration to secondary lymphoid organs may reduce the likelihood of immune rejection following transplantation. Based on this information, the current study hypothesized that $\mathrm{HO}-1$ upregulation in $\mathrm{KCs}$ may stabilize the MC membrane, decrease MC degranulation and prevent DC migration to secondary lymphoid organs, and subsequently prevent immune rejection.

\section{Materials and methods}

Animals. The experimental protocol was approved by the institutional animal ethics committee of Shanghai Tenth People Hospital of Tong Ji University (Shanghai, China).

A total of 18 male C57BL/6 mice, 8-10-weeks-old, were purchased from Schleck Experimental Animals Co. (Shanghai, China). All mice were housed in a pathogen-free facility, maintained at $26^{\circ} \mathrm{C}$ under $12 \mathrm{~h}$ light/dark cycle and had access to food and water ad libitum. They were used in accordance with the National Institutes of Health Guide for the Care and Use of Laboratory Animals of the Chinese Academy of Sciences (5).

Antibodies and reagents. KIT proto-oncogene receptor tyrosine kinase (CD117)-fluorescein isothiocyanate (FITC) (dilution 1:200; cat. no. 48-1171-80; eBioscience, Inc., San Diego, CA, USA), anti-mouse F4/80-allophycocyanin (APC) (dilution 1:20; cat. no. 47-4801-80; eBioscience, Inc.), anti-mouse CD11b-FITC (dilution 1:40; cat. no. 47-0118-41; eBioscience, Inc.), Fc fragment of IgE receptor Ia (FCeRI $\alpha$ ) -phycoerythrin (PE) (dilution 1:10; cat. no. ab124529; Abcam, Cambridge, UK) and anti-mouse CCR7 (dilution 1:200; cat. no. 25-1971-63; eBioscience, Inc.) antibodies were used. The metalloporphyrins, hemin (an HO-1 inducer) and zinc protoporphyrin (Znpp; an HO-1 inhibitor), were purchased from Enzo Life Sciences, Inc. (Farmingdale, NY, USA). Sodium cromoglicate (Sigma-Aldrich; Merck Millipore, Darmstadt, Germany) $\beta$-actin (1:2,000; cat. no. A2228; Sigma-Aldrich; Merck Millipore) and antibody against HO-1 (1:1,000; cat. no. ab13248; Abcam).

Cell preparation. C57/BL6 mice were sacrificed by anesthesia with intraperitoneal injection of ketamine (90 mg/kg) (Sigma-Aldrich; Merck Millipore) and xylazine (10 mg/kg) (Sigma-Aldrich; Merck Millipore) solution and test for loss of reflexes to ensure deep narcotization. Then, non-parenchymal cell suspensions were acquired from C57/BL6 mice using in situ collagenase perfusion of liver and $\mathrm{KCs}$ were isolated by sedimentation in a two-step Percoll gradient with selective adherence of cells to plastic flasks as previously described (3). Cell viability was determined by trypan blue exclusion, and the purity of the $\mathrm{KC}$ fraction was determined using anti-mouse F4/80-APC (dilution 1:20) and anti-mouse CD11b-FITC antibodies (dilution 1:40). Murine bone marrow-derived mast cells (BMMCs) and DCs (BMDCs) were obtained as described previously $(17,18)$. The cells were collected $8 \times 10^{5}$ and centrifuged at $135 \times \mathrm{g} 5 \mathrm{~min}$ at $4^{\circ} \mathrm{C}$, then the cells were resuspended in PBS and $2 \%$ fetal bovine serum $200 \mu \mathrm{l}$. All cells were incubated with the antibody for $30 \mathrm{~min}$ on ice, then washed twice with PBS and 2\% serum and centrifuged at 1,200 $\mathrm{x} g$ for 5 min, analysis was performed using FlowJo 7.6. The purity of BMMCs was assessed by measuring the expression of CD117 and FCeRI $\alpha$ using flow cytometry. BMMCs were used at a purity of $95 \%$. The purity of DCs was analyzed by measuring CD11c expression using flow cytometry.

$R T-P C R$ and $R T-q P C R$. Total RNA was isolated from KCs using TRIzol (Sigma-Aldrich; Merck Millipore) according to standard procedures. Thereafter, $2 \mu \mathrm{g}$ of total RNA was reverse transcribed to cDNA using the Superscript III Transcription kit (Invitrogen; Thermo Fisher Scientific, Inc., Waltham, MA, USA) with Genomic DNA Eraser (Takara Biotechnology Co., Ltd., Dalian, China) according to manufacturer's protocol. PCR was performed on a Px2 Thermal Cycler, using the following conditions: 1 cycle of denaturation at $95^{\circ} \mathrm{C}$ for $5 \mathrm{~min}, 35$ cycles of denaturation at $94^{\circ} \mathrm{C}$ for $30 \mathrm{sec}$, annealing at $55^{\circ} \mathrm{C}$ for $30 \mathrm{sec}$, and extension at $72^{\circ} \mathrm{C}$ for $40 \mathrm{sec}$ and an additional cycle of extension at $72^{\circ} \mathrm{C}$ for $10 \mathrm{~min}$. Then $2 \%$ gel was used. GAPDH was used as an internal control. qPCR was performed on a Chromo4 Four-Color Real-Time PCR Detection system (Bio-Rad Laboratories, Inc., Hercules, CA, USA) using the SYBR Premix Ex Taq II (Tli RNaseH Plus) kit (Takara Biotechnology Co., Ltd.). Using the following conditions: Initial the cycle of denaturation at $95^{\circ} \mathrm{C}$ for $30 \mathrm{sec}$, followed by 40 cycles of $95^{\circ} \mathrm{C}$ for $5 \mathrm{sec}$, annealing $60^{\circ} \mathrm{C}$ for $30 \mathrm{sec}$ and extension $70^{\circ} \mathrm{C}$ for $15 \mathrm{sec}$. Data was normalized using the $2^{-\triangle \Delta C q}$ method (19). PCR was performed on an ABI Prism 7700 (Applied Biosystems; Thermo Fisher Scientific, Inc.). For linear amplification, GAPDH was used as an internal control. The following PCR primers were synthesized by Shanghai Sangon Biological Engineering Technology \& Services Co., Ltd. (Shanghai, China): HO-1, 5'-ACGCATATA CCCGCTACCTG-3' (forward) and 5'-TGCTGATCTGGG ATTTTCCT-3' (reverse); GAPDH, 5'-TCCCTCAAGATTGTC AGCAA-3' (forward) and 5'-AGATCCACAACGGATACA TT-3' (reverse).

Protein extraction and western blotting. Reagents were purchased from Sigma-Aldrich unless otherwise indicated. Proteins were extracted from KCs and western blotting was performed. Briefly, 2.5-6x $10^{6}$ cells were incubated for $15 \mathrm{~min}$ on ice in lysis buffer [50 mM Tris $\mathrm{HCl} \mathrm{pH} 8.0 ; 120 \mathrm{mM}$ $\mathrm{NaCl} ; 0.25 \%$ Nonidet P40; $0.1 \%$ SDS; and protease inhibitors phenylmethylsulfonyl fluoride, aprotinin, leupeptin, and pepstatin (Roche Diagnostics) at a final concentration of $10 \mathrm{ng} / \mathrm{ml}, 1 \mathrm{mM}$ DTT]. A total of $60 \mathrm{mg}$, of each protein sample was subjected to $15 \%$ SDS-PAGE and blotted onto a nitrocellulose membrane (GE Healthcare Life Sciences, Chalfont, UK). The protein quantification performed using a BCA kit according to the manufacturer's protocol (Sigma-Aldrich; Merck Millipore). Membranes were blocked with $5 \%$ non-fat dry milk in TBS-Tween $(0.5 \%) 4{ }^{\circ} \mathrm{C}$ overnight and probed with either rabbit anti-mouse $\mathrm{HO}-1$ monoclonal antibody $(2 \mathrm{mg} / \mathrm{ml}$; Abcam) or rabbit anti-mouse $\beta$-actin monoclonal antibody $(1: 2,000)$ followed by horseradish peroxidase-conjugated anti-rabbit IgG antibody $(1: 3,000$; cat. no. RPN4301; GE Healthcare Life Sciences, Logan, UT, USA). Immunoreactive protein bands incubated with the enhanced chemiluminescence (ECL) reagent $30 \mathrm{sec}$ 
according to the manufacturer's protocol using an ECL detection kit (cat. no. RPN998; GE Healthcare Life Sciences) and were visualized with image lab 4.0 software.

MC degranulation assay. After treating KCs with PBS, dimethyl sulfoxide (DMSO), $50 \mu \mathrm{M} / 1$ hemin or $50 \mu \mathrm{M} / 1 \mathrm{Znpp}$ for $8 \mathrm{~h}$, cells were collected and cultured in 24-well cell culture plates at a density of $2.5 \times 10^{5}$ cells per $200 \mu \mathrm{l}$, either with direct contact with MCs or without MCs $\left(5 \times 10^{5}\right.$ cells), separated by a Transwell chamber $0.4 \mu \mathrm{m}$. The $50 \mu \mathrm{M} / 1$ sodium cromoglicate was used to pretreat the $\mathrm{MC}$ as the stabilization control. After $24 \mathrm{~h}$, each group of MCs were pre-incubated with anti-dinitrophenol (DNP)-IgE (100 ng/ml) (1:1,000, cat. no. D8406; Sigma-Aldrich; Merck Millipore) for $24 \mathrm{~h}$ and subsequently challenged using $100 \mathrm{ng} / \mathrm{ml}$ dinitrophenol-human serum albumin DNP-HSA. After $1 \mathrm{~h}$, the cell supernatant of the co-culture system was collected. Following solubilization with $0.5 \%$ Triton X-100 in Tyrode's buffer, the enzymatic activity of $\beta$-hexosaminidase in supernatants and cell pellets was measured using $\mathrm{p}$-nitrophenyl- $\mathrm{N}$-acetyl- $\beta$-D-glucosaminide in $0.1 \mathrm{M}$ sodium citrate, $\mathrm{pH} 4.5$, at $37^{\circ} \mathrm{C}$ for $60 \mathrm{~min}$. The reaction was halted by addition of $0.2 \mathrm{M}$ glycine $(\mathrm{pH} 10.7)$ and the amount of p-nitrophenol released was measured by absorbance at a wavelength of $405 \mathrm{~nm}$ using a spectrophotometer. The extent of degranulation was calculated as the p-nitrophenol absorbance of the supernatant/the total absorbance of the supernatant and detergent-solubilized cell pellet.

Analysis of pretreated MC-DC interaction and DC migration. MCs were cultured with KCs in $10 \%$ serum that were pretreated with PBS, DMSO, hemin or Znpp $24 \mathrm{~h}$, using $50 \mu \mathrm{M} / 1$ sodium cromoglicate to pretreat the MCs as the stabilization control, then MC degranulation was stimulated with anti-DNP-IgE associated with DNP-HSA. DC migration was assessed using Transwell assays ( $8 \mu \mathrm{m}$ pore size). DCs were incubated in $10 \%$ serum in upper chambers at a density of $5 \times 10^{5}$ cells per well, MCs by pretreated KCs in the lower chamber. After $48 \mathrm{~h}$, cells in the upper chamber were discarded and DCs that had migrated to the lower chamber harvested. Cells in the lower chamber were fixed with $10 \%$ methyl alcohol, stained with $0.1 \%$ crystal violet for $20 \mathrm{~min}$, washed with double distilled $\mathrm{H}_{2} \mathrm{O}$ and counted under an inverted microscope, at a magnification of $\mathrm{x} 200$.

Additionally, DCs were cultured with stimulated MCs for $24 \mathrm{~h}$ in Transwell plates Supernatants were harvested from lower chamber of the co-culture system, and prostaglandin E2 (PGE2) (cat. no. H6-KA0324; eBioscience USA), C-C motif chemokine ligand 19 (CCL-19) (cat. no. RK-KOA0264; eBioscience USA) and CCL21 (cat. no. 85-88-58214-22; eBioscience USA) were quantified by ELISA (eBioscience, Inc.) according to the manufacturer's instructions. DCs were incubated with immunofluorescent CD16/31 (dilution, $3 \mu 1$ for $10^{6}$ cells) and anti-mouse CCR7 antibodies for $30 \mathrm{~min}$ on ice then washed twice. Flow cytometry was performed using Flow Jo 7.6 software.

Statistical analysis. All statistical analysis was carried out using SPSS 9.13 (SPSS, Inc., Chicago, IL, USA) and results are presented as the mean \pm standard deviation. One-way analysis of variance along with Dunnett's post hoc test was performed to determine the statistical significance of the data. $\mathrm{P}<0.05$ was considered to indicate a statistically significant difference.

\section{Results}

Hemin induces HO-1 mRNA and protein expression in KCs. HO-1 mRNA and protein levels in KCs cultured with PBS, DMSO, hemin or Znpp were measured by RT-PCR, RT-qPCR and western blotting. HO-1 mRNA was significantly increased $8 \mathrm{~h}$ after exposure to hemin compared with incubation with PBS, DMSO or Znpp (Fig. 1). Consistent with these results, western blot analysis demonstrated that HO-1 protein levels were lower in PBS, DMSO and Znpp-treated groups after $8 \mathrm{~h}$, compared with the hemin-treated group (Fig. 2).

Upregulation of HO-1 expression in KCs may inhibit mast cell degranulation. KCs were pretreated with PBS, DMSO, hemin or Znpp for $8 \mathrm{~h}$ and cultured either in contact with MCs or separately (Fig. 3). After 24 h, MC degranulation was stimulated with anti-DNP-IgE and DNP-HSA, and enzymatic activity of $\beta$-hexosaminidase was used to estimate the level of MC degranulation usually. Following treatment with hemin, a decrease in $\beta$-hexosaminidase release was observed in $\mathrm{KCs}$ that were in contact with MCs, and also those that were separate from MCs. There was no difference between the sodium cromoglicate group and hemin group demonstrating that upregulation of HO-1 in KCs may inhibit MC degranulation and stabilize $\mathrm{MC}$ membranes.

$M C$ degranulation stimulates $C C R 7$ expression on the $D C$ surface, and stabilizing the MC membrane diminishes CCR7 expression. DC migration from peripheral tissues to secondary immune organs, particularly lymph nodes, is a prerequisite for initiating an effective immune response, and CCR7 expression at the surface of DCs facilitates homing $(20,21)$. MC membranes were stabilized by hemin-pretreated KCs, whether cultured in contact or separately as mentioned above, therefore MCs cultured separately were selected for use in further experiments. Degranulation was stimulated and they were co-cultured with DCs. After $24 \mathrm{~h}$, expression of CCR7 at the DC surface was decreased in hemin and sodium cromoglicate-treated groups compared with PBS, DMSO and Znpp-treated groups (Fig. 4), and there was no difference between the sodium cromoglicate group and hemin group.

MC degranulation stimulates DC migration. Transwell plates were used to investigate DC migration (Fig. 5), and five areas were chosen randomly for cell counting under the inverted microscope at magnification of $\times 200$. Pretreatment of KCs with hemin (Fig. 5D) upregulated HO-1, stabilized MC membranes and decreased migration of DCs to the lower Transwell chamber, compared with KCs pretreated with PBS (Fig. 5B), DMSO (Fig. 5C) or Znpp (Fig. 5E). Again, there was no difference between the sodium cromoglicate group (Fig. 5F) and hemin group, confirming that $\mathrm{MC}$ degranulation stimulated DC migration.

MC degranulation stimulates DC migration via release of PGE2, CCL19 and CCL21. Stimulation of MC degranulation 
A

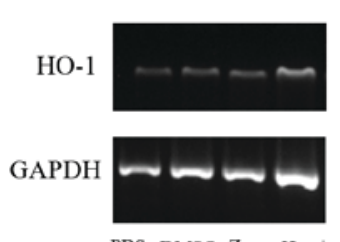

B

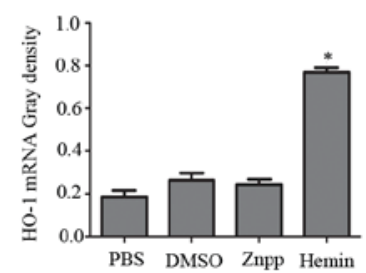

$\mathrm{C}$

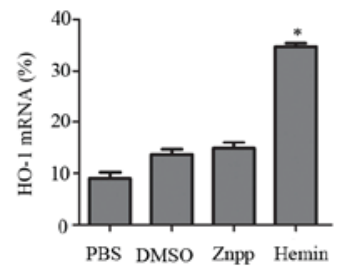

Figure 1. HO-1 mRNA expression in Kupffer cells. (A) HO-1 was evaluated by RT-PCR following pretreatment with PBS, DMSO, Znpp or hemin (50 $\mu$ M). Expression of HO-1 was increased in the hemin group compared with the other treatment groups. (B) Bands corresponding to HO-1 and GAPDH were quantified densitometrically. "P<0.05 Hemin group vs. Znpp group. (C) HO-1 was evaluated by RT-qPCR following pretreatment. HO-1 levels were normalized to GAPDH. "P<0.05 Hemin group vs. Znpp group. HO-1, heme oxygenase-1; RT-PCR, reverse transcription-polymerase chain reaction; DMSO, dimethyl sulfoxide; Znpp, zinc protoporphyrin; RT-qPCR, reverse transcription-quantitative PCR.

A

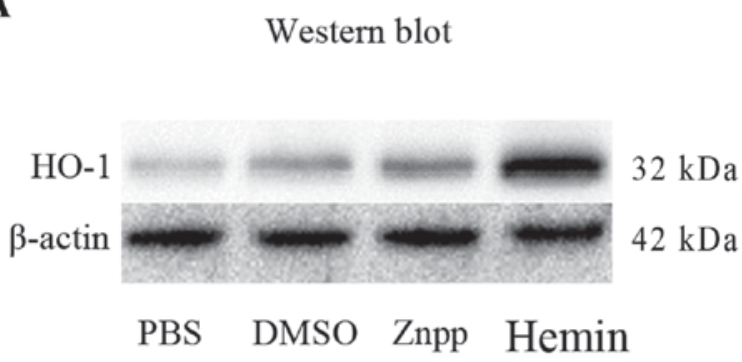

B

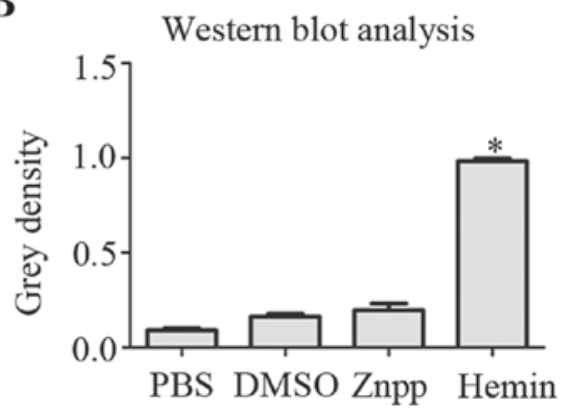

Figure 2. HO-1 protein levels in Kupffer cells. (A) Protein levels were evaluated by western blotting following pretreatment with PBS, DMSO, Znpp or hemin $(50 \mu \mathrm{M}) . \beta$-actin was used as an internal control. (B) Bands corresponding to HO-1 and $\beta$-actin protein were quantified densitometrically. Results are expressed as the ratio of full-length HO-1 to $\beta$-actin. " $\mathrm{P}<0.05$ Hemin group vs. Znpp group. HO-1, heme oxygenase-1; DMSO, dimethyl sulfoxide; Znpp, zinc protoporphyrin.

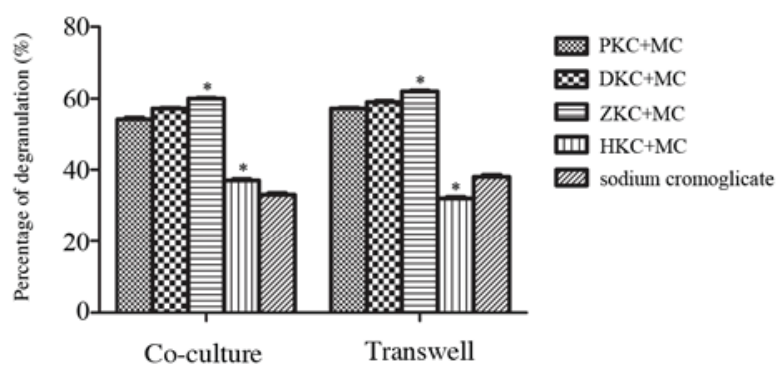

Figure 3. Effect of heme oxygenase-1 upregulation in KCs on MC degranulation. KCs were pretreated with PBS, dimethyl sulfoxide, hemin or zinc protoporphyrin for $8 \mathrm{~h}$ prior to interaction with MCs. MC degranulation induced by monoclonal anti-DNP-IgE and DNP-HSA was evaluated using 4-nitrophenyl N-acetyl- $\beta$-D-glucosaminide. $\mathrm{PKC}+\mathrm{MC}, \mathrm{DKC}+\mathrm{MC}$ and $\mathrm{ZKC}+\mathrm{MC}$ groups elicited $\mathrm{MC}$ degranulation, whereas the $\mathrm{HKC}+\mathrm{MC}$ and sodium cromoglicate groups inhibited the $\mathrm{MC}$ degranulation. The $\mathrm{HKC}+\mathrm{MC}$ and sodium cromoglicate groups were not significantly different. Similar results were observed whether $\mathrm{KCs}$ and $\mathrm{MCs}$ were in contact or separated by a Transwell. ${ }^{\mathrm{P}}<0.05 \mathrm{HKC}+\mathrm{MC}$ group vs. $\mathrm{ZKC}+\mathrm{MC}$ group. $\mathrm{KC}$, Kupffer cells; PKC, PBS-treated KCs; DKCs, dimethyl sulfoxide-treated KCs; ZKCs, zinc protoporphyrin-treated $\mathrm{KCs}$; HKCs, hemin-treated $\mathrm{KCs}$; MC, mast cell; DNP-HSA, dinitrophenol-human serum albumin.

may result in the release of cytokines that influence DC migration. The levels cytokines PGE2, CCL19 and CCL21 were measured in supernatants from co-cultures using ELISA (Fig. 6). Compared with membrane-stabilized MCs, represented by hemin and sodium cromoglicate-treated groups, degranulated MCs produced significantly increased levels of all three cytokines. Due to the fact that increased MC degranulation led to increased DC migration, these cytokines therefore potentially contributed to the changes observed in DC migration.

\section{Discussion}

HO-1 catabolizes heme into carbon monoxide, biliverdin and free iron, which helps to protect cells against a variety of potential oxidative stimuli (22). Recent studies have demonstrated that HO-1 may confer a protective effect in organ transplantation, since HO-1 and its byproducts may protect the allograft from IRI and the immune response following a liver transplant (23). In rat liver, HO-1 is highly expressed in KCs (24), liver-resident macrophages that have an important role in the acute and chronic responses of the liver to toxic compounds. Our previous study demonstrated that preconditioning donor liver with nodosin perfusion reduces IRI in rats, and this occurs via upregulation of HO-1 that may then prime $\mathrm{KCs}$, which go on to suppress the immune response (25). The understanding of the role of KCs in IRI and the immune response is incomplete. Upregulation of HO-1 may alleviate IRI and decrease MC degranulation, whereas increased MC degranulation promoted IRI in rat liver (12). MCs are known to produce various factors responsible for the allergic response, including histamine and inflammatory proteins (26). These cells function in the innate (27) and adaptive immune system (28). MCs 

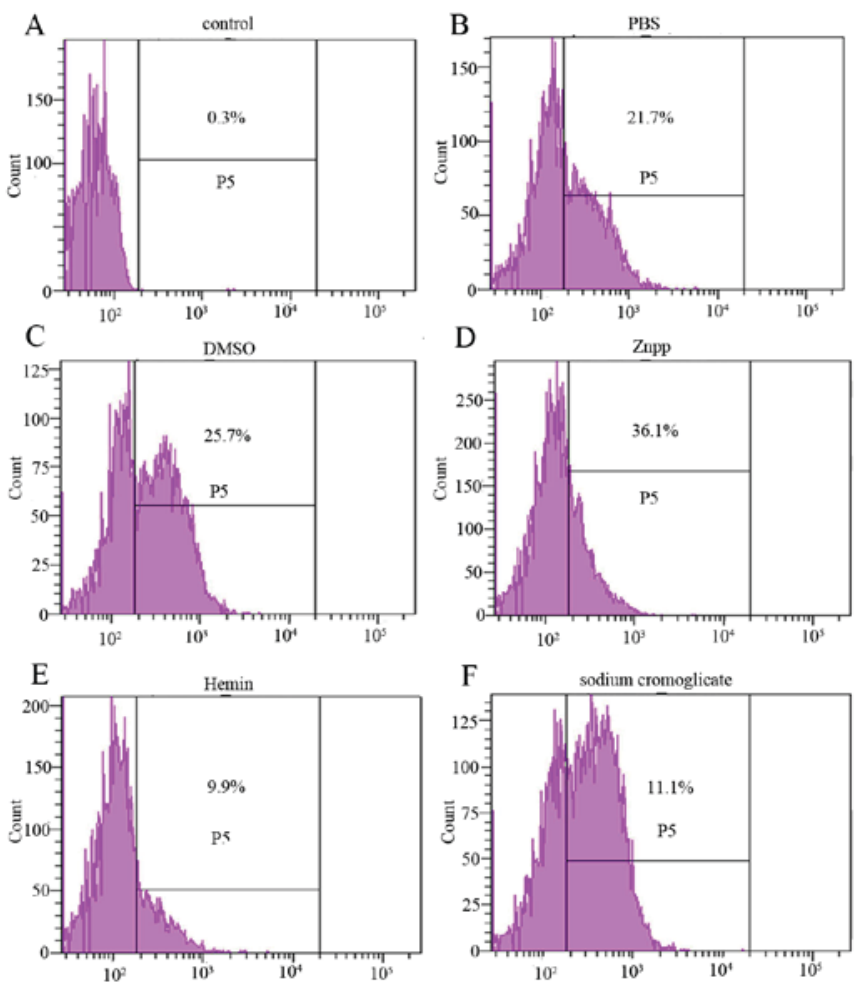

G

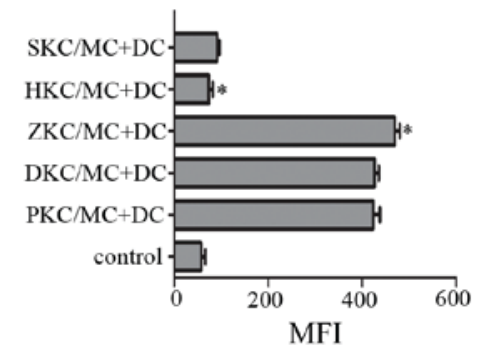

Figure 4. Expression of chemokine receptor CCR7 on the surface of DCs. Levels of CCR7 on DCs, that were co-cultured with activated MCs interacting with KCs that had been pretreated with (A) control, (B) PBS, (C) DMSO, (D) Znpp, (E) hemin and (F) sodium cromoglicate, were detected. Expression of CCR7 on the DC surface was decreased by hemin and sodium cromoglicate. $(\mathrm{G})$ Expression of CCR7 on the DC surface is presented as MFI, which was measured by flow cytometry after $24 \mathrm{~h}$ of incubation (with isotype control subtracted). Data are presented as the percentage MFI of control group, which was set as $100 \%$. ${ }^{*} \mathrm{P}<0.05 \mathrm{HKC} / \mathrm{MC}+\mathrm{DC}$ group vs. ZKC/MC+DC group. DCs, dendritic cells; MCs, mast cells; KCs, Kupffer cells; DMSO, dimethyl sulfoxide; Znpp, zinc protoporphyrin; MFI, mean fluorescence intensity; SKC, sodium cromoglicate-treated KCs; HKC, hemin-treated KCs; ZKC, Znpp-treated KCs; DKC, DMSO-treated KCs; PKC, PBS-treated KCs; CCR7, C-C motif chemokine receptor 7.

may release cytokines that influence the diseased state, and inhibition of MC degranulation by HO-1 disrupted DC maturation in vitro (29). This indicates that for MCs to perform their function in the adaptive immune system, DC maturation and migration may be required. DCs reside in an immature state in peripheral blood and tissues until activated by inflammatory cytokines or antigens. Following activation, DCs are transported via the afferent lymphatic system into the draining lymph node before initiating an immune response (30). DC migration is influenced by CCRs on the cell surface, and CCR7 is the most important receptor in numerous diseases (31). Pahne-Zeppenfeld et al (32) reported that cervical cancer cells suppress the induction of CCR7 in
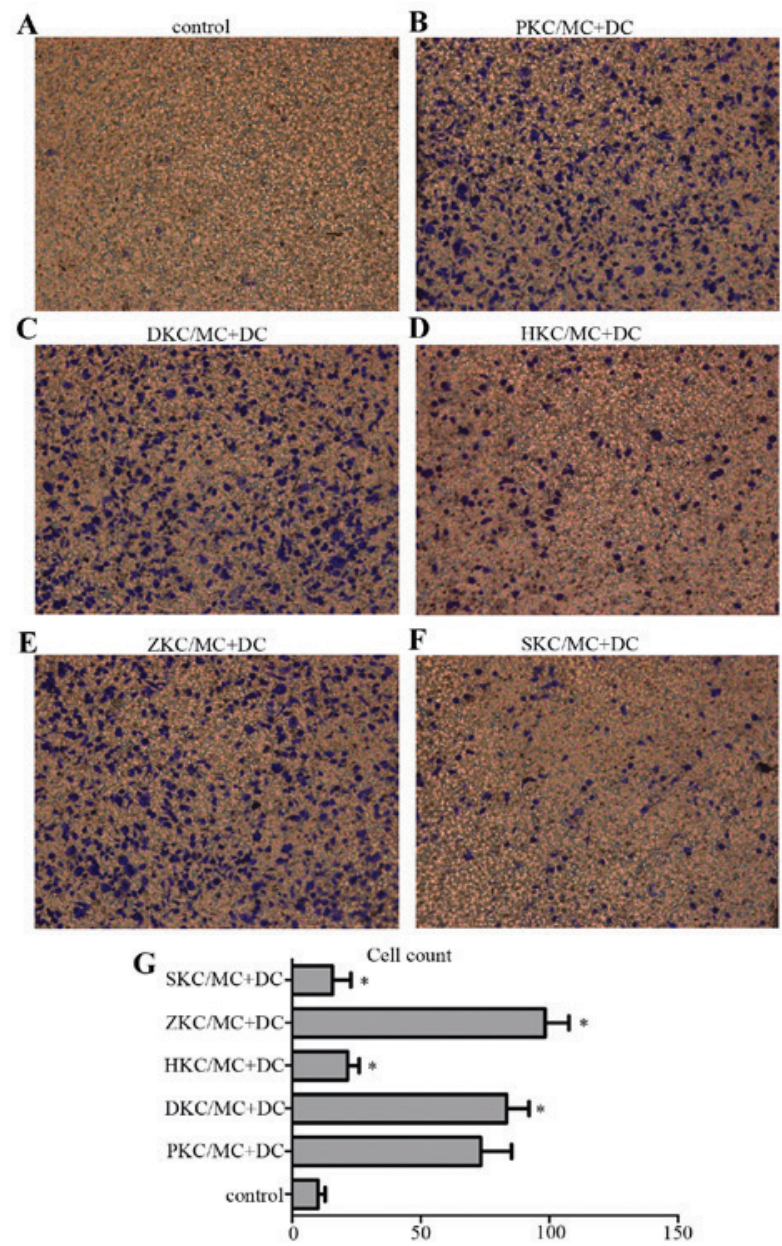

Figure 5. DC migration in vitro. DC migration was tested using a chemotaxis assay in a 24-well Transwell chamber. Lower wells were loaded with activated MCs interacting with pretreated KCs or medium only (control), and upper wells were loaded with $5 \times 10^{5} \mathrm{DCs} /$ well/condition and incubated for $48 \mathrm{~h}$ at $37^{\circ} \mathrm{C}$. The cell number was $80-100 /$ field for (B:PKC/MC+DC), (C:DKC/MC+DC) and (E:ZKC/MC+DC), and 10-30/field for (A:control), (D:HKC/MC+DC) and (F:SKC/MC+DC). (G) Presents the number of cells in each group. (n=5/group). " $\mathrm{P}<0.05$ vs. control. $\mathrm{DC}$, dendritic cells; $\mathrm{MC}$, mast cells; KC, Kupffer cells; SKC, sodium cromoglicate-treated KCs; HKC, hemin-treated KCs; ZKC, zinc protoporphyrin-treated KCs; DKC, dimethyl sulfoxide-treated KCs; PKC, PBS-treated KCs.

phenotypically mature DCs, which impairs their migration toward the lymph node that is required for the adaptive immune response. CCR7 and its ligands, CCL19 and CCL21, control a diverse array of migratory events during adaptive immunity (33). Blocking CCR7 or its ligands was effective in promoting graft survival in animal models of heart or islet allotransplantation (34). Expression of CCR7 is influenced by PGE2, and PGE2 antagonists downregulate CCR7 expression $(35,36)$. Torres et al $(37)$ determined that MC degranulation caused PGE2 release, which inhibited asthma.

Our previous study revealed that the upregulation the HO-1 expression of liver tissue may inhibit MC degranulation and the $\mathrm{HO}-1$ expression in the $\mathrm{KC}$ was intracellular. In the present study $\mathrm{HO}-1$ was upregulated in $\mathrm{KCs}$ when they were pretreated with hemin, then co-cultured with $\mathrm{MC}$, the MC membranes were stabilized. Co-culturing DCs with membrane-stabilized MCs resulted in downregulation of CCR7 on the surface of DCs. Furthermore, the levels of the cytokines PGE2, CCL9 

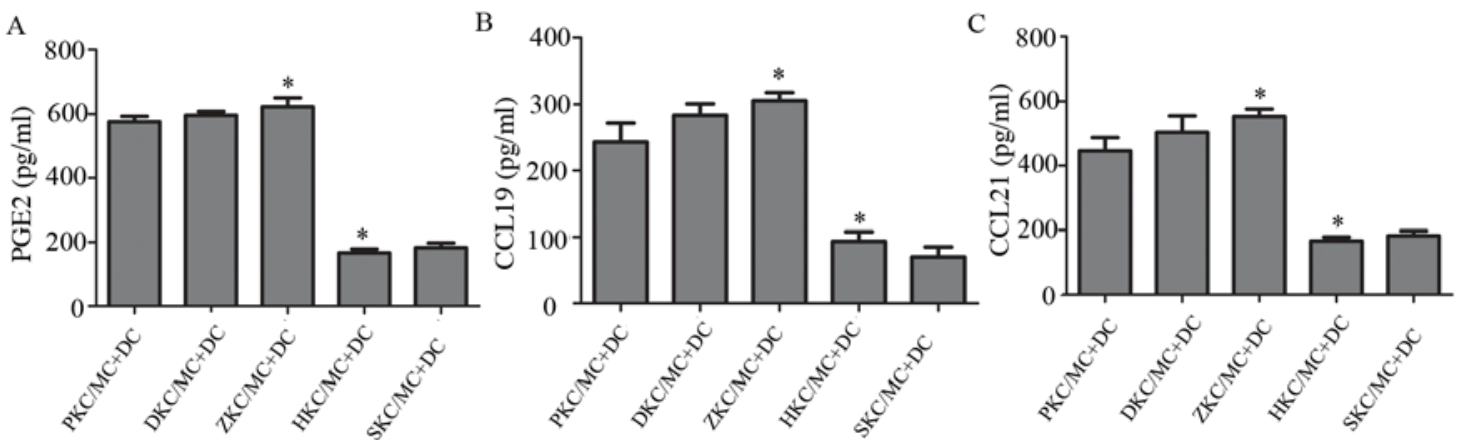

Figure 6. Changes in cytokine levels in cell supernatants. (A) PGE2, (B) CCL19 and (C) CCL21 levels in the supernatant of co-cultured cells of each group. "P<0.05 ZKC/MC+DC vs. HKC/MC+DC. PGE2, prostaglandin E2; CCL, C-C motif chemokine ligand; DC, dendritic cells; MC, mast cells; KC, Kupffer cells; SKC, sodium cromoglicate-treated KCs; HKC, hemin-treated KCs; ZKC, zinc protoporphyrin-treated KCs; DKC, dimethyl sulfoxide-treated KCs; PKC, PBS-treated KCs.

and CCL21 were decreased in the supernatants of co-cultured DCs. Membrane-stabilized MCs also impaired DC migration. The present study demonstrates a potential mechanism of DC homing in vitro and may explain a possible mechanism that MC degranulation would induce immune rejection. The relevance of this potential mechanism in vivo requires further investigation.

\section{Acknowledgements}

National Natural Science Foundation of China (grant nos. 81270555; 81470897 and 81472501) and Program for New Century Excellent Talents in University (grant no. NECT-13-0422).

\section{References}

1. Waller HL, Harper SJ, Hosgood SA, Bagul A, Kay MD, Kaushik M, Yang B, Bicknell GR and Nicholson ML: Differential expression of cytoprotective and apoptotic genes in an ischaemia-reperfusion isolated organ perfusion model of the transplanted kidney. Transplant Int 20: 625-631, 2007.

2. Ke BB, Buelow R, Shen XD, Melinek J, Amersi F, Gao F, Ritter T, Volk HD, Busuttil RW and Kupiec-Weglinski JW: Hemeoxygenase 1 gene transfer prevents CD95/Fas ligand-mediated apoptosis and improves liver allograft survival via carbon monoxide signaling pathway. Hum Gene Ther 13: 1189-1199, 2002.

3. Zeng Z, Huang HF, Chen MQ, Song F and Zhang YJ: Heme oxy-genase-1 protects donor livers from ischemia/reperfusion injury: The role of Kupffer cells. World J Gastroenterol 16 : 1285-1292, 2010.

4. Yue LH, Zhao YL, Chen J and Lu DR: Effect of fusion protein TAT and heme oxygenase-1 on liver sinusoidal endothelial cells apoptosis during preservation injury. Chin Med J (Engl) 123 $68-73,2010$.

5. Jung ID, Lee JS, Lee CM, Noh KT, Jeong YI, Park WS, Chun SH, Jeong SK, Park JW, Son KH, et al: Induction of indoleamine 2,3-dioxygenase expression via heme oxygenase-1-dependant pathway during murine dendritic cell maturation. Biochem Pharmacol 80: 491-505, 2010.

6. Thomson AW and Knolle PA: Antigen-presenting cell function in the tolerogenic liver environment. Nat Rev Immunol 11: 753-766, 2010

7. Wang CF, Wang ZY, Tao SF, Ding J, Sun LJ, Li JY and Quan ZW: Preconditioning donor liver with Nodosin perfusion lessens rat ischemia reperfusion injury via heme oxygenase-1 upregulation. J Gastroenterol Hepatol 27: 832-840, 2012.

8. de Vries VC, Wasiuk A, Bennett KA, Benson MJ, Elgueta R, Waldschmidt TJ and Noelle RJ: Mast cell degranulation breaks peripheral tolerance. Am J Transplant 9: 2270-2280, 2009.
9. Iwasaki W, Kume M, Kudo K, Uchinami H, Kikuchi I, Nakagawa Y, Yoshioka M and Yamamoto Y: Changes in the fatty acid composition of the liver with the administration of $\mathrm{N}-3$ polyunsaturated fatty acids and the effects on warm ischemia/reperfusion injury in the rat liver. Shock 3: 306-314, 2010.

10. Vliagoftis $\mathrm{H}$ and Befus AD: Mast cells at mucosal frontiers. Curr Mol Med 6: 573-589, 2005.

11. Choi AM and Alam J: Heme oxygenase-1: Function, regulation, and implication of a novel stress-inducible protein in oxidant-induced lung injury. Am J Respir Cell Mol Biol 15: 9-19, 1996.

12. Yang MQ, Ma YY, Tao SF, Ding J, Rao LH, Jiang H and Li JY: Mast cell degranulation promotes ischemia ereperfusion injury in rat liver. J Surg Res 186: 170-178, 2014

13. Takamiya R, Murakami M, Kajimura M, Goda N, Makino N, Takamiya Y, Yamaguchi T, Ishimura Y, Hozumi $\mathrm{N}$ and Suematsu M: Stabilization of mast cells by heme oxygenase-1: An anti-inflammatory role. Am J Physiol Heart Circ Physiol 3: H861-H870, 2002.

14. Bakdash G, Schneider LP, van Capel TM, Kapsenberg ML, Teunissen MB and de Jong EC: Intradermal application of vitamin D3 increases migration of CD14+ dermal dendritic cells and promotes the development of Foxp3+ Regulatory T cells. Hum Vaccin Immunother 2: 250-258, 2013.

15. Chen M, Huang L, Shabier Z and Wang J: Regulation of the lifespan in dendritic cell subsets. Mol Immunol 44: 2558-2565, 2007.

16. Schaeuble K, Hauser MA, Rippl AV, Bruderer R, Otero C, Groettrup $M$ and Legler DF: Ubiquitylation of the chemokine receptor CCR7 enables efficient receptor recycling and cell migration. J Cell Sci 125: 4463-4474, 2012.

17. Kageyama-Yahara N, Suehiro Y, Yamamoto T and Kadowaki M IgE-induced degranulation of mucosal mast cells is negatively regulated via nicotinic acetylcholine receptors. Biochem Biophys Res Commun 377: 321-325, 2008.

18. Cho NH, Cheong TC, Min JH, Wu JH, Lee SJ, Kim D, Yang JS, Kim S, Kim YK and Seong SY: A multifunctional core-shell nanoparticle for dendritic cell-based cancer immunotherapy. Nat Nanotechnol 6: 675-682, 2011.

19. Livak KJ and Schmittgen TD: Analysis of relative gene expression data using real-time quantitative PCR and the 2(-Delta Delta C(T)) method. Methods 25: 402-408, 2001.

20. MartIn-Fontecha A, Sebastiani S, Höpken UE, Uguccioni M, Lipp M, Lanzavecchia A and Sallusto F: Regulation of dendritic cell migration to the draining lymph node: Impact on T lymphocyte traffic and priming. J Exp Med 198: 615-621, 2003.

21. Verdijk P, Aarntzen EH, Punt CJ, de Vries IJ and Figdor CG: Maximizing dendritic cell migration in cancer immunotherapy. Expert Opin Biol Ther 8: 865-874, 2008.

22. Katana E, Skoura L, Giakoustidis D, Takoudas D, Malisiovas N and Daniilidis M: Association between the heme oxygenase-1 promoter polymorphism and renal transplantation outcome in Greece. Transplant Proc 42: 2479-2485, 2010.

23. Salahudeen AA, Jenkins JK, Huang H, Ndebele K and Salahudeen AK: Overexpression of heme oxygenase protects renal tubular cells against cold storage injury: Studies using hemin induction and HO-1 gene transfer. Transplantation 72: $1498-1504,2001$ 
24. Tamura T, Kondo T, Ogawa K, Fukunaga K and Ohkohchi N: Protective effect of heme oxygenase-1 on hepatic ischemia-reperfusion injury through inhibition of platelet adhesion to the sinusoids. J Gastroenterol Hepatol 28: 700-706, 2013.

25. Zhang M, Xu S, Han Y and Cao X: Apoptotic cells attenuate fulminant hepatitis by priming Kupffer cells to produce interleukin-10 through membrane-bound TGF- $\beta$. Hepatology 53: 306-316, 2011.

26. Beaven MA: Our perception of the mast cell from Paul Ehrlich to now. Eur J Immuno 39: 11-25, 2009

27. Leslie M: Mast cells show their might. Science 317: 614-616, 2007.

28. McLachlan JB, Hart JP, Pizzo SV, Shelburne CP, Staats HF, Gunn MD and Abraham SN: Mast cell-derived tumor necrosis factor induces hypertrophy of draining lymph nodes during infection. Nat Immunol 4: 1199-1205, 2003.

29. Ma YY, Yang MQ, Wang CF, Ding J and Li JY: Inhibiting mast cell degranulation by $\mathrm{HO}-1$ affects dendritic cell maturation in vitro. Inflamm Res 63: 527-537, 2014

30. Gouwy M, Struyf S, Leutenez L, Pörtner N, Sozzani S and Van Damme J: Chemokines and other GPCR ligands synergize in receptor-mediated migration of monocyte-derived immature and mature dendritic cells. Immunobiology 219: 218-229, 2014.

31. Förster R, Davalos-Misslitz AC and Rot A: CCR7 and its ligands: Balancing immunity and tolerance. Nat Rev Immunol 8: 362-371, 2008.
32. Pahne-Zeppenfeld J, Schröer N, Walch-Rückheim B, Oldak M, Gorter A, Hegde S and Smola S: Cervical cancer cell-derived interleukin-6 impairs CCR7-de pendent migration of MMP-9-e xpressingDendritic cells. Int J Cancer 134: 2061-2073, 2014

33. Comerford I, Harata-Lee Y, Bunting MD, Gregor C, Kara EE and McColl SR: A myriad of functions and complex regulation of the CCR7/CCL19/CCL21 chemokine axis in the adaptive immune system. Cytokine Growth Factor Rev 24: 269-283, 2013.

34. Ziegler E, Gueler F, Rong S, Mengel M, Witzke O, Kribben A, Haller H, Kunzendorf U and Krautwald S: CL19-IgG prevents allograft rejection by impairment of immune cell trafficking. J Am Soc Nephrol 17: 2521-2532, 2006.

35. Luft T, Jefford M,Luetjens P, Toy T, Hochrein H, Masterman KA, Maliszewski C, Shortman K, Cebon J and Maraskovsky E: Functionally distinct dendritic cell (DC) populations induced by physiologic stimuli: Prostaglandin E(2) regulates the migratory capacity of specific DC subsets. Blood 100: 1362-1372, 2002.

36. Pan MR, Hou MF, Chang HC and Hung WC: Cyclooxygenase-2 up-regulates CCR7 via EP2/EP4 receptor signaling pathways to enhance lymphatic invasion of breast cancer cells. J Biol Chem 283: 11155-11163, 2008.

37. Torres R, Picado C and de Mora F: The PGE2-EP2-mast cell axis: An antiasthma mechanism. Mol Immunol 63: 61-68, 2015. 